\title{
Research on the ground bearing capacity of sulfate saline soil
}

\author{
Zheng Yu-xin, Li chun-yan \\ (Xinjiang Vocational and Technical College of Communications, Xinjinag Urumqi 831402 ) \\ (Xinjiang Industy Technical College ,Xinjinag Urumqi 830021)
}

KEY WORDS: salinesoil ; sulfate ground; bearing capacity

ABSTRACT: According to salt expansion and strength parameter index $\mathrm{c}$ and $\varphi$ of sulphate salty soil, we assume that three-layer soil body of salty soil is destroyed. Assuming the network slippage diagram and using rigid-plasticity balance theorem, the calculation method of bearing capacity in salty soil area is obtained.

\section{Foreword}

Salt in soil is unevenly distributed, thus mechanical properties of soil are different. In the calculation of bearing capacity of foundation soil, if soil body is isotropic body, it is not realistic to mechanically use Terzaghi K. Formula. considering that saline soil in salty soil is defined in the vertical section with $1-2 \mathrm{~m}$ of depth in the earth's surface. Dissolving salt content is greater than $1 \%$ of land. Salty soil under $2 \mathrm{~m}$ of the earth's surface is affected by the capillary action of underground water and evaporation. Salinity is migrated to the range and it has the most significant accumulative phenomenon, so that there is a large difference in shear strength parameters between this soil layer and the soil layer with the less salinity.

\section{Slippage network diagram of the salty soil}

Salt expansion generally takes place in 40-60m of the surface layer. Mechanical properties of soil body in the salt expansion are greatly reduced. Relevant data show that $50 \%$ of salt in salty soil is gathered in $50 \mathrm{~cm}$ of the earth's surface, the foundation can be divided into three layers: the top layer is the double salt layer, while the next layer is the soil layer with the less salt. The bottom layer is the soil layer without salt or less salt. The shear strength index in the first layer is $\mathrm{c} 1$ and $\varphi 1$; that of the second layer is c2 and $\varphi$. The bottom layer is considered as the homogenous soil layer. And the shear strength index is $\mathrm{c} 3$ and $\varphi 3$.assuming that soil body destruction refers to overall shear failure form. Based on the internal friction angle $\varphi$ of soil, flow net method is used to determine the similar slippage network diagram. Next, the ultimate balance theory is used to deduce the bearing capacity of foundation soil formula that is suitable for salty soil.

Tthe embankment is considered the foundation without the buried depth. The width can be obtained by using the cross section area of the highway(as shown in Figure 1). According to the three-layer assumption of salty soil body and flow net method principles, the stress circle method is used to similarly solve the slippage network of soil body, as shown in Figure 2. The thickness of total salt bed is assumed as $h 1+h 2(h 1+h 2 \leq 200 \mathrm{~cm})$. the salt bed thickness of salt expansion is $\mathrm{h} 1(\mathrm{~h} 1 \leq 60 \mathrm{~cm})$. The basic width is $\mathrm{b}$ and basic buried depth is $\mathrm{d}$. 


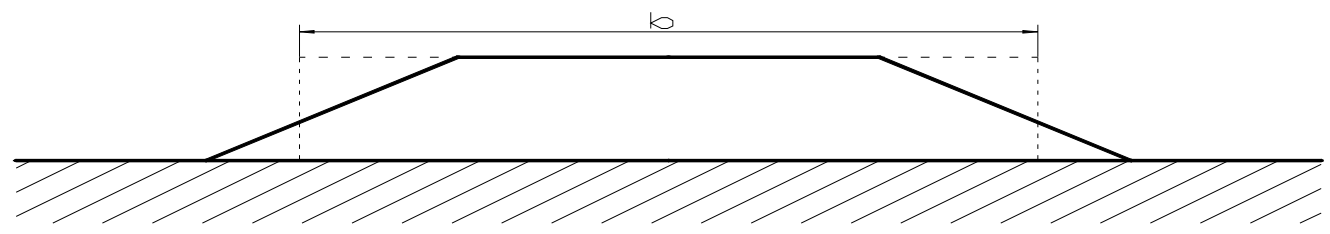

Fig.1 Equivalent Conversion of Basic Conversion

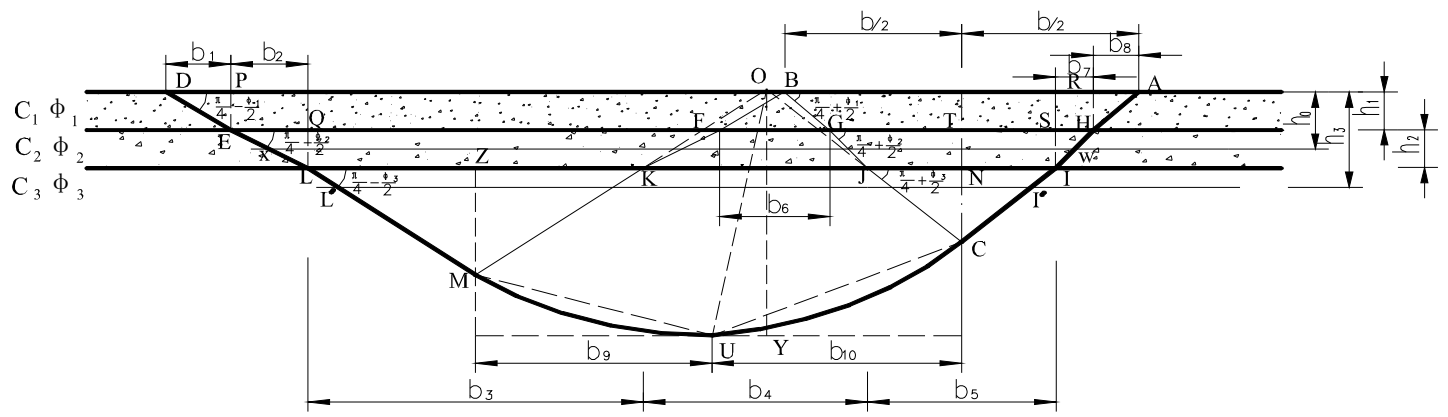

Fig.2 Network Slippage Diagram;

\section{The foundation calculation of bearing capacity for salty soil}

In the calculation of bearing capacity, it can be observed that bearing capacity of foundation soil in the salty soil area is composed of the ultimate bearing capacity p1 generated by cohesive force and ultimate bearing capacity p2 generated by soil body's dead load. Ultimate bearing capacity generated by both of them is respectively calculated as:

\section{Ultimate bearing capacity 1 generated by cohesive force $c$}

As shown in Figure 2, cohesive force in the first and second layers is respectively c1 and c2. Internal friction angle is $\varphi 1$ and $\varphi 2$. Assuming that downward cohesive force along the horizontal line $\mathrm{BD}$ has the vertical even load, the value is $\cot \cot \varphi 1$. The downward vertical even load on $\mathrm{AB}$ is $\mathrm{c} 1 \cot \varphi 1$. EH has the upward vertical even $\operatorname{load} c 1 \cot \varphi 1$. EF has the downward cohesive force with the vertical even load c $2 \cot \varphi 2$. Similarly, FG and GH has the downward vertical even load c $2 \cot \varphi 2$. LI has the upward vertical even load $c 2 \cot \varphi 2$.

1. Counter-force N1 and horizontal force X1 on DE are calculated by vertical even load.

Among which, vertical force on DE is $F_{1}=D E \cdot c_{1} \cot \varphi_{1}=h_{1} c_{1} \cot \varphi_{1} \cdot \cot \left(\frac{\pi}{4}-\frac{\varphi_{1}}{2}\right)$;

$N_{1}=h_{1} c_{1} \cot \varphi_{1} \cdot \cot \left(\frac{\pi}{4}-\frac{\varphi_{1}}{2}\right) \cdot \csc \left(\frac{\pi}{4}-\frac{\varphi_{1}}{2}\right) \quad X_{1}=N_{1} \cdot \cos \left(\frac{\pi}{4}-\frac{\varphi_{1}}{2}\right)=h_{1} c_{1} \cot \varphi_{1} \cdot \cot ^{2}\left(\frac{\pi}{4}-\frac{\varphi_{1}}{2}\right)$

2. Counter-force $\mathrm{N} 2$ and horizontal force $\mathrm{X} 2$ on EL are calculated by vertical even load:

Among which, vertical force on DE is $F_{2}=E L \cdot c_{2} \cot \varphi_{2}=h_{2} c_{2} \cot \varphi_{2} \cdot \cot \left(\frac{\pi}{4}-\frac{\varphi_{2}}{2}\right)$;

$X_{2}=N_{2} \cdot \cos \left(\frac{\pi}{4}-\frac{\varphi_{2}}{2}\right)=h_{2} c_{2} \cot \varphi_{2} \cdot \cot ^{2}\left(\frac{\pi}{4}-\frac{\varphi_{2}}{2}\right) \quad N_{2}=h_{2} c_{2} \cot \varphi_{2} \cdot \cot \left(\frac{\pi}{4}-\frac{\varphi_{2}}{2}\right) \cdot \csc \left(\frac{\pi}{4}-\frac{\varphi_{2}}{2}\right) ;$

3. By using cohesive force $\mathrm{c}$ on the undersoil, viscous force T3, counter-force N3 and horizontal force X3 on LM are calculated. Among which, viscous force on $\mathrm{LM}$ was $T_{3}=L M \cdot c_{3}=N_{3}$; 
$L M=M K=r_{1}-b_{4} \cdot \cos \left(\frac{\pi}{4}-\frac{\varphi_{3}}{2}\right) \quad X_{3}=T_{3} \cdot \cos \left(\frac{\pi}{4}-\frac{\varphi_{3}}{2}\right)+N_{3} \cdot \cos \left(\frac{\pi}{4}-\frac{\varphi_{3}}{2}\right)=2 \cos \left(\frac{\pi}{4}-\frac{\varphi_{3}}{2}\right) \cdot L M \cdot c_{3}$

4. Calculate horizontal viscous force $\mathrm{X} 4$ on arc JK section

$$
\begin{aligned}
& X_{4}=J K \cdot c_{3}=\left(b_{4}+\frac{1}{2} b_{3}+\frac{1}{2} b_{5}\right) \cdot c_{3} \\
& =\left\{h_{1}\left[\cot \left(\frac{\pi}{4}-\frac{\varphi_{1}}{2}\right)+\cot \left(\frac{\pi}{4}+\frac{\varphi_{1}}{2}\right)\right]+h_{2}\left[\cot \left(\frac{\pi}{4}-\frac{\varphi_{2}}{2}\right)+\cot \left(\frac{\pi}{4}+\frac{\varphi_{2}}{2}\right)\right]+\right. \\
& \left\{\left\{h_{1}\left[\cot \left(\frac{\pi}{4}-\frac{\varphi_{1}}{2}\right)+\cot \left(\frac{\pi}{4}+\frac{\varphi_{1}}{2}\right)\right]+h_{2} \cot \left(\frac{\pi}{4}-\frac{\varphi_{2}}{2}\right)+h_{2} \cdot \cot \left(\frac{\pi}{4}+\frac{\varphi_{2}}{2}\right)\right.\right. \\
& \left.+\left[\frac{1}{2} b-h_{2} \cot \left(\frac{\pi}{4}+\frac{\varphi_{2}}{2}\right)-h_{1} \cot \left(\frac{\pi}{4}+\frac{\varphi_{1}}{2}\right)\right]\right\} \cdot \sec \left(\frac{\pi}{4}+\frac{\varphi_{3}}{2}\right) \cdot e^{\frac{\pi}{2} \tan \varphi_{3}} \\
& \left.-\left\{h_{1}\left[\cot \left(\frac{\pi}{4}-\frac{\varphi_{1}}{2}\right)+\cot \left(\frac{\pi}{4}+\frac{\varphi_{1}}{2}\right)\right]+h_{2}\left[\cot \left(\frac{\pi}{4}-\frac{\varphi_{2}}{2}\right)+\cot \left(\frac{\pi}{4}+\frac{\varphi_{2}}{2}\right)\right]\right\} \cdot \cos \left(\frac{\pi}{4}-\frac{\varphi_{3}}{2}\right)\right\} \\
& \left.\cdot \cos \left(\frac{\pi}{4}-\frac{\varphi_{3}}{2}\right)+\frac{b}{2}-\left[h_{2} \cot \left(\frac{\pi}{4}+\frac{\varphi_{2}}{2}\right)+h_{1} \cot \left(\frac{\pi}{4}+\frac{\varphi_{1}}{2}\right)\right]\right\} \cdot c_{3} ;
\end{aligned}
$$

5. Calculate the even load p1 on the basic AB and calculate counter-force N5 and horizontal force $\mathrm{X} 5$ on $\mathrm{AH} . \quad N_{5}=A R \cdot\left(c_{1} \cdot \cot \varphi_{1}+p_{1}\right) \cdot \csc \left(\frac{\pi}{4}+\frac{\varphi_{1}}{2}\right) \quad X_{5}=N_{5} \cdot \cos \left(\frac{\pi}{4}+\frac{\varphi_{1}}{2}\right)$

6. Calculate viscous force T6, counter-force N6 and horizontal force X6 on HI.

Among which, vertical force on $\mathrm{SH}$ is $F_{6}=S H \cdot\left(p_{1}+c_{2} \cot \varphi_{2}\right)$;

$$
S H=b_{7}=h_{2} \cot \left(\frac{\pi}{4}+\frac{\varphi_{2}}{2}\right) ; \quad X_{6}=N_{6} \cdot \cos \left(\frac{\pi}{4}+\frac{\varphi_{2}}{2}\right)=h_{2}\left(p_{1}+c_{2} \cot \varphi_{2}\right) \cot ^{2}\left(\frac{\pi}{4}+\frac{\varphi_{2}}{2}\right)
$$

7. Under the action of even load p1, calculate viscous force T7, counter-force N7 and horizontal force on IC. $X_{7}=N_{7} \cos \left(\frac{\pi}{4}+\frac{\varphi_{2}}{2}\right)$

When horizontal force of soil body on the slippage network curve is known, according to rigid-plasticity body of ultimate balance theory, force on the horizontal direction keeps balance. The balance equation is constructed: $\quad X_{1}+X_{2}+X_{3}+X_{4}=X_{5}+X_{6}+X_{7}$

Based on formula(1), it can obtain $p_{1}=f_{1}\left(c_{1}, \varphi_{1}, c_{2}, \varphi_{2}, c_{3}, \varphi_{3}\right)$

\section{Ultimate bearing capacity p2 generated by dead load of the soil body}

As calculating the bearing capacity of foundation soil, it gets involved in lots of influence factors. This study should consider the shear strength index, unit weight, basic width and basic buried depth. Unit weight of sol is also affected by the underground water level, in addition to relate to soil variety. As a result, influences of unit weight changes on bearing capacity of foundation soil should be considered in the calculation process. Here, two situations should be considered: one is $2 \mathrm{~m}$ of the underground water level from the earth's surface, assuming that distance is $\mathrm{h} 0$; the other one is more than $2 \mathrm{~m}$ of the underground water level from the earth's surface, assuming that the distance is $\mathrm{h} 0$ '.

(1) Set up the distance from the underground water level to the earth's surface is h0; unit capacity of soil body on the upper saline soil is $\mathrm{y} 01$; the unit capacity of soil capacity on the second layer saline soil is y02; the partial unit capacity under the underground water level is y2; the unit capacity of soil body under the lower layer is $\mathrm{y} 3$.

1. Under the action of dead load, calculate counter-force P1 and horizontal force Y1 on DE Among which, soil body on De is $\frac{1}{2} r_{01} h_{1}{ }^{2} \cdot \cot \left(\frac{\pi}{4}-\frac{\varphi_{1}}{2}\right)$, thus 


$$
P_{1}=\frac{1}{2} r_{01} h_{1}^{2} \cdot \cot \left(\frac{\pi}{4}-\frac{\varphi_{1}}{2}\right) \cdot \csc \left(\frac{\pi}{4}-\frac{\varphi_{1}}{2}\right) \quad Y_{1}=P_{1} \cdot \cos \left(\frac{\pi}{4}-\frac{\varphi_{1}}{2}\right)=\frac{1}{2} r_{01} h_{1}^{2} \cdot \cot ^{2}\left(\frac{\pi}{4}-\frac{\varphi_{1}}{2}\right)
$$

2. Calculate counter-force P2 and P3, and horizontal force Y2 and Y3 on EX and XL.

Among which, soil body on EX is $\left(h_{0}-h_{1}\right) \cdot \cot \left(\frac{\pi}{4}-\frac{\varphi_{2}}{2}\right) \cdot\left[r_{01} h_{1}+\frac{1}{2} r_{02}\left(h_{0}-h_{1}\right)\right]$, thus

$$
\begin{aligned}
& P_{2}=\left[r_{01} h_{1}\left(h_{0}-h_{1}\right)+\frac{1}{2} r_{02}\left(h_{0}-h_{1}\right)^{2}\right] \cot \left(\frac{\pi}{4}-\frac{\varphi_{2}}{2}\right) \cdot \csc \left(\frac{\pi}{4}-\frac{\varphi_{2}}{2}\right) \\
& Y_{2}=\left[r_{01} h_{1}\left(h_{0}-h_{1}\right)+\frac{1}{2} r_{02}\left(h_{0}-h_{1}\right)^{2}\right] \cot ^{2}\left(\frac{\pi}{4}-\frac{\varphi_{2}}{2}\right)
\end{aligned}
$$

Soil body on XL is:

$$
\begin{aligned}
& \left(h_{1}+h_{2}-h_{0}\right) \cdot \cot \left(\frac{\pi}{4}-\frac{\varphi_{2}}{2}\right) \cdot\left[r_{01} h_{1}+r_{02}\left(h_{0}-h_{1}\right)+\frac{1}{2} r_{2}\left(h_{1}+h_{2}-h_{0}\right)\right], \text { thus } \\
& P_{3}=\left(h_{1}+h_{2}-h_{0}\right)\left[r_{01} h_{1}+r_{02}\left(h_{0}-h_{1}\right)+\frac{1}{2} r_{2}\left(h_{1}+h_{2}-h_{0}\right)\right] \cot \left(\frac{\pi}{4}-\frac{\varphi_{2}}{2}\right) \cdot \csc \left(\frac{\pi}{4}-\frac{\varphi_{2}}{2}\right) \\
& Y_{3}=\left(h_{1}+h_{2}-h_{0}\right)\left[r_{01} h_{1}+r_{02}\left(h_{0}-h_{1}\right)+\frac{1}{2} r_{2}\left(h_{1}+h_{2}-h_{0}\right)\right] \cdot \cot ^{2}\left(\frac{\pi}{4}-\frac{\varphi_{2}}{2}\right)
\end{aligned}
$$

3. Calculate counter-force P4 and horizontal force Y4 on LM

Soil body on LM is $\frac{1}{2} b_{3} \cdot\left[r_{01} h_{1}+r_{02}\left(h_{0}-h_{1}\right)+r_{2}\left(h_{1}+h_{2}-h_{0}\right)+\frac{1}{4} r_{3} b_{3} \tan \left(\frac{\pi}{4}-\frac{\varphi_{3}}{2}\right)\right]$, thu

$$
\begin{aligned}
& P_{4}=\frac{1}{2} b_{3} \cdot\left[r_{01} h_{1}+r_{02}\left(h_{0}-h_{1}\right)+r_{2}\left(h_{1}+h_{2}-h_{0}\right)+\frac{1}{4} r_{3} b_{3} \tan \left(\frac{\pi}{4}-\frac{\varphi_{3}}{2}\right)\right] \cdot \csc \left(\frac{\pi}{4}-\frac{\varphi_{3}}{2}\right) \\
& Y_{4}=\frac{1}{2} b_{3} \cdot\left[r_{01} h_{1}+r_{02}\left(h_{0}-h_{1}\right)+r_{2}\left(h_{1}+h_{2}-h_{0}\right)+\frac{1}{4} r_{3} b_{3} \tan \left(\frac{\pi}{4}-\frac{\varphi_{3}}{2}\right)\right] \cdot \cot \left(\frac{\pi}{4}-\frac{\varphi_{3}}{2}\right)
\end{aligned}
$$

\section{Calculate counter-force P5 and P6 and horizontal force Y5 and Y6}

It can be observed from the network diagram that the counter-force horizontal force caused by dead load on arc MC is inconsistent by regarding the deepest point of the slip line as the boundary. In order to solve this point, the logarithmic spiral equation $r=r_{0} e^{\theta \tan \varphi}$ is applied. Assuming that the horizontal plane is the starting point of the logarithmic spiral, thus in the equation:

$$
\begin{aligned}
& r_{0}=\left\{h_{1}\left[\cot \left(\frac{\pi}{4}-\frac{\varphi_{1}}{2}\right)+\cot \left(\frac{\pi}{4}+\frac{\varphi_{1}}{2}\right)\right]+h_{2} \cot \left(\frac{\pi}{4}-\frac{\varphi_{2}}{2}\right)+h_{2} \cot \left(\frac{\pi}{4}+\frac{\varphi_{2}}{2}\right)\right. \\
& \left.+\left[\frac{1}{2} b-h_{2} \cot \left(\frac{\pi}{4}+\frac{\varphi_{2}}{2}\right)-h_{1} \cot \left(\frac{\pi}{4}+\frac{\varphi_{1}}{2}\right)\right]\right\} \cdot \sec \left(\frac{\pi}{4}+\frac{\varphi_{3}}{2}\right) e^{-\left(\frac{\pi}{4}+\frac{\varphi_{3}}{2} \tan \varphi\right.}
\end{aligned}
$$

Soil weight on the arc MU is:

$$
\begin{aligned}
b_{9}\left[r_{01} h_{1}+r_{02}\left(h_{0}-h_{1}\right)+r_{2}\left(h_{1}+h_{2}-h_{0}\right)+\frac{1}{2} b_{3} \tan \left(\frac{\pi}{4}-\frac{\varphi_{3}}{2}\right)+\frac{1}{2} h_{3}\right] \\
P_{5}=\left\{b_{9}\left[r_{01} h_{1}+r_{02}\left(h_{0}-h_{1}\right)+r_{2}\left(h_{1}+h_{2}-h_{0}\right)+\frac{1}{2} b_{3} \tan \left(\frac{\pi}{4}-\frac{\varphi_{3}}{2}\right)+\frac{1}{2} h_{3}\right]\right\} \csc \theta_{1} \\
Y_{5}=\left\{b_{9}\left[r_{01} h_{1}+r_{02}\left(h_{0}-h_{1}\right)+r_{2}\left(h_{1}+h_{2}-h_{0}\right)+\frac{1}{2} b_{3} \tan \left(\frac{\pi}{4}-\frac{\varphi_{3}}{2}\right)+\frac{1}{2} h_{3}\right]\right\} \cot \theta_{1}
\end{aligned}
$$

Soil weight on the arc CU is: $\quad b_{10}\left[r_{01} h_{1}+r_{02}\left(h_{0}-h_{1}\right)+r_{2}\left(h_{1}+h_{2}-h_{0}\right)+\frac{1}{2} b_{5} \tan \left(\frac{\pi}{4}+\frac{\varphi_{3}}{2}\right)+\frac{1}{2} h_{4}\right]$

$$
\begin{gathered}
P_{6}=\left\{b_{10}\left[r_{01} h_{1}+r_{02}\left(h_{0}-h_{1}\right)+r_{2}\left(h_{1}+h_{2}-h_{0}\right)+\frac{1}{2} b_{5} \tan \left(\frac{\pi}{4}+\frac{\varphi_{3}}{2}\right)+\frac{1}{2} h_{4}\right]\right\} \csc \theta_{2} \\
Y_{6}=\left\{b_{10}\left[r_{01} h_{1}+r_{02}\left(h_{0}-h_{1}\right)+r_{2}\left(h_{1}+h_{2}-h_{0}\right)+\frac{1}{2} b_{5} \tan \left(\frac{\pi}{4}+\frac{\varphi_{3}}{2}\right)+\frac{1}{2} h_{4}\right]\right\} \cot \theta_{2}
\end{gathered}
$$


5. Under the action of even load P2 on the dead load of soil body, calculate counter-force P7 and horizontal force $\mathrm{Y} 7$ on $\mathrm{AH}$.

Among which, soil weight on $\mathrm{AH}$ is $h_{1} \cot \left(\frac{\pi}{4}+\frac{\varphi_{1}}{2}\right)\left(p_{2}+\frac{1}{2} r_{01} h_{1}\right)$, thus $P_{7}=h_{1}\left(p_{2}+\frac{1}{2} r_{01} h_{1}\right) \cot \left(\frac{\pi}{4}+\frac{\varphi_{1}}{2}\right) \cdot \csc \left(\frac{\pi}{4}+\frac{\varphi_{1}}{2}\right) \quad Y_{7}=h_{1}\left(p_{2}+\frac{1}{2} r_{01} h_{1}\right) \cot ^{2}\left(\frac{\pi}{4}+\frac{\varphi_{1}}{2}\right)$

6. Calculate counter-force P8 and P9 and horizontal force $\mathrm{Y} 8$ and $\mathrm{Y} 9$ on $\mathrm{HW}$ and WI. Among which, soil weight on HW is $\left(h_{0}-h_{1}\right) \cdot \cot \left(\frac{\pi}{4}+\frac{\varphi_{2}}{2}\right) \cdot\left[r_{01} h_{1}+\frac{1}{2} r_{02}\left(h_{0}-h_{1}\right)+p_{2}\right]$, thus $Y_{8}=\left(h_{0}-h_{1}\right)\left[r_{01} h_{1}+\frac{1}{2} r_{02}\left(h_{0}-h_{1}\right)+p_{2}\right] \cdot \cot ^{2}\left(\frac{\pi}{4}+\frac{\varphi_{2}}{2}\right)$

Soil weight on WI is $\left(h_{1}+h_{2}-h_{0}\right) \cdot \cot \left(\frac{\pi}{4}+\frac{\varphi_{2}}{2}\right) \cdot\left[r_{01} h_{1}+r_{02}\left(h_{0}-h_{1}\right)+\frac{1}{2} r_{2}\left(h_{1}+h_{2}-h_{0}\right)\right]$, thus $P_{9}=\left(h_{1}+h_{2}-h_{0}\right)\left[r_{01} h_{1}+r_{02}\left(h_{0}-h_{1}\right)+\frac{1}{2} r_{2}\left(h_{1}+h_{2}-h_{0}\right)\right] \cot \left(\frac{\pi}{4}+\frac{\varphi_{2}}{2}\right) \cdot \csc \left(\frac{\pi}{4}+\frac{\varphi_{2}}{2}\right)$ $Y_{9}=\left(h_{1}+h_{2}-h_{0}\right)\left[r_{01} h_{1}+r_{02}\left(h_{0}-h_{1}\right)+\frac{1}{2} r_{2}\left(h_{1}+h_{2}-h_{0}\right)\right] \cdot \cot ^{2}\left(\frac{\pi}{4}+\frac{\varphi_{2}}{2}\right)$

7. Calculate counter-force P10 and horizontal force Y10 on IC.

The soil weight on IC is $\frac{1}{2} b_{5}\left[r_{01} h_{1}+r_{02}\left(h_{0}-h_{1}\right)+r_{2}\left(h_{1}+h_{2}-h_{0}\right)+\frac{1}{2} b_{5} \tan \left(\frac{\pi}{4}+\frac{\varphi_{3}}{2}\right)\right]$, thus $P_{10}=\frac{1}{2} b_{5}\left[r_{01} h_{1}+r_{02}\left(h_{0}-h_{1}\right)+r_{2}\left(h_{1}+h_{2}-h_{0}\right)+\frac{1}{2} b_{5} \tan \left(\frac{\pi}{4}+\frac{\varphi_{3}}{2}\right)\right] \cdot \csc \left(\frac{\pi}{4}+\frac{\varphi_{3}}{2}\right)$ $Y_{10}=\frac{1}{2} b_{5} \cdot\left[r_{01} h_{1}+r_{02}\left(h_{0}-h_{1}\right)+r_{2}\left(h_{1}+h_{2}-h_{0}\right)+\frac{1}{2} b_{5} \tan \left(\frac{\pi}{4}+\frac{\varphi_{3}}{2}\right)\right] \cdot \cot \left(\frac{\pi}{4}+\frac{\varphi_{3}}{2}\right)$

Considering the balance of force on the horizontal direction, the equilibrium equation is established as:

$\mathrm{Y}_{1}+\mathrm{Y}_{2}+\mathrm{Y}_{3}+\mathrm{Y}_{4}+\mathrm{Y}_{5}=\mathrm{Y}_{6}+\mathrm{Y}_{7}+\mathrm{Y}_{8}+\mathrm{Y}_{9}+\mathrm{Y}_{10}$

According to the formula(5-5), it can obtain: $p_{2}=f_{2}\left(c_{1}, \varphi_{1}, c_{2}, \varphi_{2}, c_{3}, \varphi_{3}\right)$.

By combining with formula(5-4) and formula(5-5), it can obtain:

$$
p_{u}=p_{1}+p_{2}=f_{1}\left(c_{1}, \varphi_{1}, c_{2}, \varphi_{2}, c_{3}, \varphi_{3}\right)+f_{2}\left(c_{1}, \varphi_{1}, c_{2}, \varphi_{2}, c_{3}, \varphi_{3}\right)
$$

When underground water level is under $2 \mathrm{~m}$ salt bed, the calculation method of ultimate bearing capacity 2 caused by soil weight is similar to the above-mentioned method.

\section{Reference}

[1] Zheng Yuxin. Xinjiang Gan- Mo highway saline soil compaction test research [D], Railway Construction,2013, 23(2) 140 142

[2] Zheng Yuxin. Eolan-sand In xinjiang Highway Deformations Of Saline Disease Control Application [J]. Traffic standardization,2013(24):94-97.

[3] Zheng Yuxin. Eolian-sand Subgrade Construction Technology Research In A-He Desert Highway [J] .Hunan Communication Science and Technology, 2015(1):36-39

[4] Zheng Yuxin. Subgrade resist deformation grouting plan in goaf of coal field [J]. Subgrade Engineering, 2011 (5):130-132. 